\title{
Lithium and s-process enrichment in massive galactic AGB stars
}

\author{
P. García-Lario
}

ISO Data Centre, Apartado de Correos 50727, Villafranca del Castillo, E-28080 Madrid, Spain

F. D'Antona

Osservatorio Astronomico di Roma, I-00040 Monteporzio, Italy

J. Lub

Sterrewacht Leiden, Postbus 9513, NL-2300 RA Leiden, The Netherlands

B. Plez

Astronomiska Observatoriet, Box 515, S-75120 Uppsala, Sweden

Atomspektroskopi, Fysiska Institution, Box 118, S-22100 Lund, Sweden

H.J. Habing

Sterrewacht Leiden, Postbus 9513, NL-2300 RA Leiden, The Netherlands

\begin{abstract}
We present the first results of a wide observational program intended to study the effects of hot-bottom burning and of the third dredge-up in the chemical evolution of the most luminous AGB stars in our Galaxy. The main goal is to provide new observational constraints to the models through the analysis of lithium and s-process element abundances in a carefully selected sample of galactic AGB stars. Our results indicate that, unlike observed in the Magellanic Clouds, the most massive AGB candidates in our Galaxy do not show any significant enrichment in s-process elements. In addition, only some of them are found to be Li-rich.
\end{abstract}

\section{Introduction}

Studies of AGB stars in the Magellanic Clouds (Plez et al. 1993; Smith et al. 1995) show a lack of high-luminosity $\mathrm{C}$ stars beyond the limit of $\mathrm{M}_{b o l} \sim-6$. This has been interpreted as the consequence of the transformation of ${ }^{12} \mathrm{C}$ into ${ }^{14} \mathrm{~N}$ via the $\mathrm{CN}$ cycle in massive AGB stars, where the temperature of the convective envelope is hot enough ( $\mathrm{T} \geq 2 \times 10^{7} \mathrm{~K}$ ) (Sackmann \& Boothroyd 1992; Wood et al. 1983). These hot-bottom burning (HBB) models also predict the production of ${ }^{7} \mathrm{Li}$, through the ${ }^{7}$ Be transport mechanism (Cameron \& Fowler 1971), which 


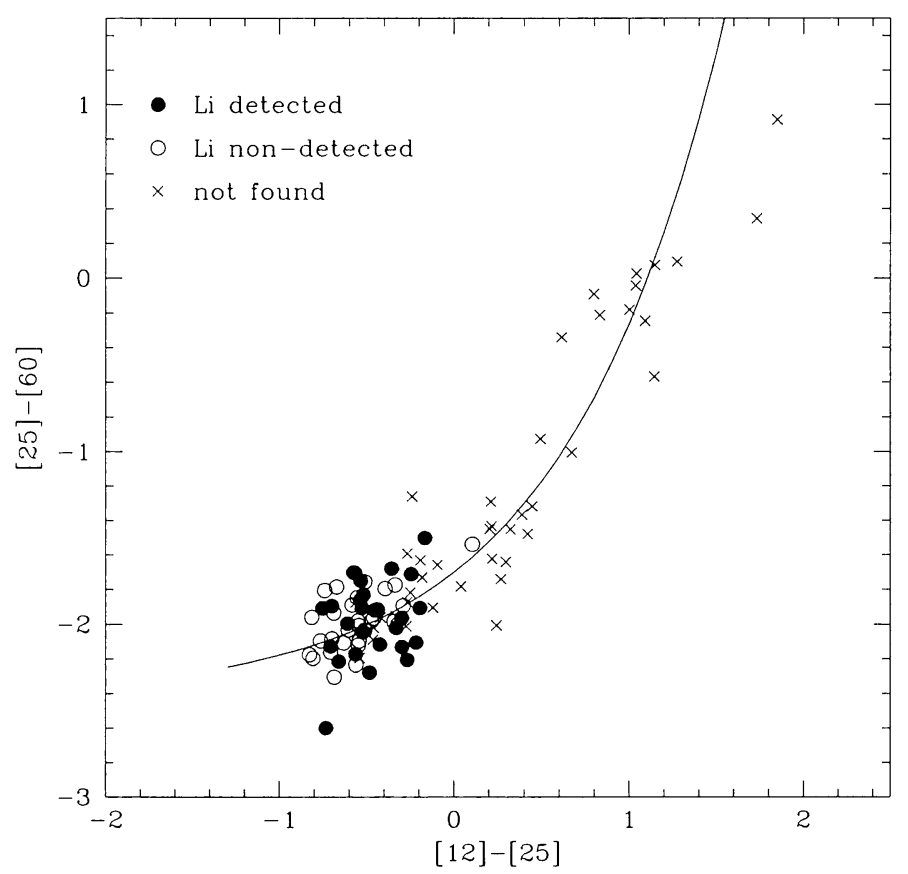

Figure 1. Distribution of the sources included in our sample in the IRAS two-colour diagram [12]-[25] vs. [25]-[60].

should be detectable for a short time in the atmosphere of the star, together with the s-process heavy elements formed by the capture of the neutrons produced during the thermal pulses, via the so called third dredge-up.

Extensive surveys have been carried out searching for lithium in AGB stars in the Magellanic Clouds (Smith \& Lambert 1990; Smith et al. 1995) resulting in the detection of strong Li lines only in luminous O-rich AGB stars in the range $-7 \leq \mathrm{M}_{b o l} \leq-6$. These stars are also enriched in s-process elements, as expected.

Inside our Galaxy, only a handful of Li-rich stars have been found (Abia et al. 1993) and, unlike those detected in the Magellanic Clouds, they are not so luminous. Some of them are intermediate mass C-stars and S-stars with a modest luminosity. Recent model calculations show that a large Li abundance is expected only for the most massive AGB stars (4 to $7 \mathrm{M}_{\odot}$ ) (D'Antona \& Mazzitelli 1996) which, in addition, should be O-rich. However, an observational problem exists with these stars. Most of them experience a strong mass loss and they are soon heavily obscured by thick circumstellar envelopes, making optical observations very difficult.

Here we present the first qualitative results of a wide observational programme based on high-resolution optical spectroscopy centered on a carefully selected sample of strongly reddened galactic O-rich AGB stars. The main goal is to search for the presence of lithium and s-process elements in the spectra of these stars, as the signature of hot-bottom burning and of the third dredge-up, respectively. The detailed abundance analysis will be presented elsewhere. 


\section{Selection of the sample}

Sources were selected from the IRAS Point Source Catalogue on the basis of their far-infrared colours and their photometric behaviour. The best candidate stars are expected to be very bright infrared sources. They should be Mira-like variables with the longest periods (sometimes more than 500 days) and very large amplitude variations (10 magnitudes in the $\mathrm{V}$ band) with a strong $\mathrm{OH}$ maser emission. Fig. 1 shows the distribution of the sources selected in the farinfrared IRAS two-colour diagram. They fall along the AGB sequence previously defined by van der Veen et al. (1989), at places which correspond to different thickness of the circumstellar envelope. They span a wide range of periods (from only 300 days up to more than 1000 days) and $\mathrm{OH}$ expansion velocities (from just a few $\mathrm{km} \mathrm{s}^{-1}$ to more than $20 \mathrm{~km} \mathrm{~s}^{-1}$ ).

Both the period and the $\mathrm{OH}$ expansion velocities have been taken as distance independent mass indicators since recent studies indicate that both the expansion velocity of circumstellar envelopes of AGB stars showing $\mathrm{OH}$ maser emission and the period of variability are correlated with the mass of the progenitor star, the group of stars with the larger expansion velocities and the longer periods showing a galactic distribution which corresponds to a more massive population (Habing et al. 1994; Sevenster et al. 1995).

\section{Observations}

High-resolution ( $R \sim 50000)$ echelle spectra in the range 5500-9000 $\AA$ were obtained at the William Herschel Telescope (La Palma, Spain) with UES and at the $3.60 \mathrm{~m}$ ESO telescope (La Silla, Chile) with CASPEC during several runs in August 1996, February 1997 and July 1997. 96 sources were tried, and useful spectra were obtained for 55 . The remaining 41 sources were either too red to obtain any significant information from the optical spectra or the counterpart was simply not found.

\section{Results}

Fig. 2 shows some of the spectra obtained in the region around the Li I line at $6707 \AA$. As we can see in this Figure, the Li I line is completely absent in some of the stars in our sample while it is found to be very strong in some others.

Table 1 displays the distribution of detections and non-detections as a function of the known periods and $\mathrm{OH}$ expansion velocities. Most of the AGB stars with the shorter periods are not detected in the Li I line while those stars with periods beyond 500 days (but still observable in the optical) are detected as Li-strong sources. Note, however, that for these objects we are very close to the limit of detection in the optical range, since the number of them too red or not found increase dramatically for periods longer than 500 days.

Similar statistics are obtained if we consider the expansion velocities derived from the $\mathrm{OH}$ maser emission. For low expansion velocities, below $6 \mathrm{~km} \mathrm{~s}^{-1}$, there are no detections, while stars showing higher expansion velocities are predominantly Li-rich. Again, the same observational problems arise when we try to 


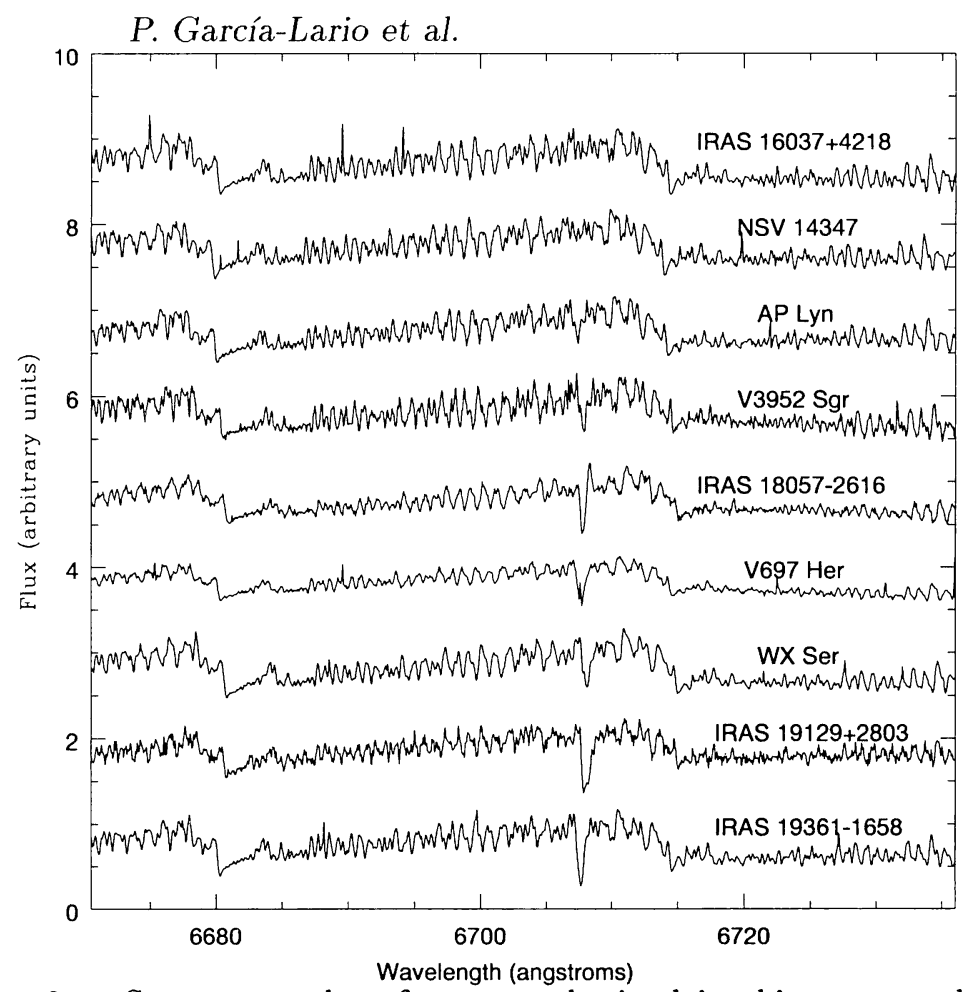

Figure 2. Some examples of spectra obtained in this survey where we show the different strengths found in the Li I $\lambda 6707 \AA$ line.

observe the stars with the highest expansion velocities, completely obscured in the optical.

If the detection of lithium is taken as a signature of HBB and the period and the expansion velocities are accepted as valid mass indicators, our results confirm that a minimum mass is needed for the activation of HBB in AGB stars in the Galaxy. As it was previously observed in the Magellanic Clouds, this explains the absence of high luminosity $\mathrm{C}$ stars also in our Galaxy. However, unlike observed in the Magellanic Clouds, there are a few AGB stars in our sample with very long periods and very high expansion velocities for which lithium is not detected.

Concerning s-process elements, we used the $\mathrm{ZrO}$ band at $6474 \AA$ as s-process enhancement indicator. Surprisingly, this band was not detected in any of the sources observed, nor the elemental lines corresponding to s-process elements in the range $7400-7600 \AA$ seem to be enhanced with respect to what it is observed in normal supergiants. Thus, we conclude that the most luminous O-rich AGB stars in our Galaxy are not S-stars, again unlike observed in the Magellanic Clouds.

\section{Discussion}

Lithium is expected to be produced in massive AGB stars as soon as the star begins the thermally pulsing phase and until ${ }^{3} \mathrm{He}$ is completely exhausted. 
Table 1. Distribution of detection/non-detections as a function of the distance-independent mass indicators used

\begin{tabular}{ccccc} 
Period (days) & Observed & Li detected & Li not detected & Not found \\
\hline $300-400$ & 11 & 1 & 9 & 1 \\
$400-500$ & 9 & 6 & 2 & 1 \\
$500-700$ & 15 & 5 & 2 & 8 \\
$>700$ & 14 & 1 & 0 & 13 \\
\hline
\end{tabular}

\begin{tabular}{ccccc}
$\mathrm{V}_{\text {exp }}\left(\mathrm{km} \mathrm{s}^{-1}\right)$ & Observed & Li detected & Li not detected & Not found \\
\hline$<6$ & 11 & 0 & 10 & 1 \\
$6-9$ & 11 & 8 & 2 & 1 \\
$9-12$ & 13 & 4 & 3 & 6 \\
$>12$ & 39 & 4 & 4 & 31 \\
\hline
\end{tabular}

Based on preliminary results derived from models recently developed by our group and which will be presented elsewhere, we obtain that for a solar metallicity and a $5-6 \mathrm{M}_{\odot}$ model, ${ }^{3} \mathrm{He}$ exhaustion takes place very rapidly as a consequence of the large temperature at the bottom of the convective envelope $\left(\sim 6010^{6} \mathrm{~K}\right)$. During the lithium production phase there is a strong enhancement of the $\mathrm{Li}$ abundance $-\log \epsilon(\mathrm{Li})=+4.0$ - but it drops very soon after a few pulses, something which could explain the non-detection of lithium in some of the most massive objects observed.

For the same metallicity and $4 \mathrm{M}_{\odot}$, the stars undergo a few pulses before the production of lithium starts and this is not so huge $-\log \epsilon(\mathrm{Li})=+2.5-3.5$ at $\mathrm{M}_{b o l}=-6$ - but it lasts for longer, decreasing again when ${ }^{3} \mathrm{He}$ is exhausted, so that the stars spend $\sim 50 \%$ of their lifetimes as Li-rich.

A stronger mass loss in our Galaxy than in the Magellanic Clouds could also explain the different results obtained. Large temperature fluctuations are expected in the bottom of the envelope if mass loss is very strong, leading to strong fluctuations of the $\mathrm{Li}$ abundance within each thermal pulse, which might be burned out during interpulses. In spite of this, although the $\mathrm{Li}$ abundance goes up and down during the TP phase, the Li-rich phase would last longer and we would expect many more Li-rich stars than Li-poor (the ratio expected would be 8 to 2 which is exactly what we find in our survey).

The assumption of a stronger mass loss in our Galaxy looks reasonable considering the number of heavily obscured AGB stars known in our Galaxy compared to those in the Magellanic Clouds (whether this is a consequence of the different metallicity deserves further investigation). This would also be consistent with the even larger number of obscured AGB stars known in the galactic bulge.

The correct identification of the stars in our sample as AGB stars (against a possible classification of them as population I supergiants) is supported by the characteristic infrared photometric properties observed. The large amplitude variations are proto-typical of stars in the AGB phase, and would be completely 
unusual for a supergiant star, expected to show only irregular luminosity fluctuations.

In order to explain the absence of s-process elements enhancements in these stars one can argue that we might be observing them still in a very early stage of development as AGB stars, especially since the sources observed are not yet strongly obscured by their circumstellar envelopes. However, this would be inconsistent with the same objects being at the end of the Li production phase, as suggested by the absence of lithium in some of them, as a consequence of ${ }^{3} \mathrm{He}$ exhaustion. Alternatively, it could be that the Li production phase has not yet even been reached by these stars.

On the other hand, massive AGB stars in our Galaxy might evolve much faster than those in the Magellanic Clouds. Thus, there would only be time for a few thermal pulses to occur during the AGB lifetime, not enough to produce a significant change in the elemental abundances observed at the surface of the star. It should be noted that, contrary to the effects of hot-bottom burning, which are immediately detectable at the surface of the star, the s-process enhancement requires some kind of dredge-up to be active and it might be observable only after a long series of thermal pulses.

If our interpretion is correct, we would expect that the less luminous AGB stars show the stronger s-process enhancements. In fact, this is what was already found for AGB stars in the Magellanic Clouds in previous works (see Figure 4 in Smith et al. 1995).

Acknowledgments. This work was financed by the Spanish Ministerio de Educación y Cultura through the project PB94-1274.

\section{References}

Abia C., Isern J., Canal R., 1993, A\&A 275, 96

Cameron A.G.W., Fowler W.A., 1971, ApJ 164, 111

D'Antona F., Mazzitelli I., 1996, ApJ 470, 1093

Habing H.J., Tignon J., Tielens A.G.G.M., 1994, A\&A 286, 523

Plez B., Smith V.V., Lambert D.L., 1993, ApJ 418, 812

Sackmann I.-J., Boothroyd A.I., 1992, ApJ 392, L71

Sevenster M.N., Dejonghe H., Habing H.J., 1995, A\&A 299, 689

Smith V.V., Lambert D.L., 1990, ApJ 361, L69

Smith V.V., Plez B., Lambert D.L. Lubowich D.A., 1995 ApJ 441, 735

van der Veen W.E.C.J., Habing H.J., Geballe T.R., 1989, A\&A 226, 108

Wood P.R., Bessell M.S., Fox M.W., 1983, ApJ 272, 99 